\title{
Crack characterization in metallic plates using vibrothermography
}

\author{
* Departamento de Física Aplicada I, Escuela Técnica Superior de Ingeniería, Universidad del País Vasco, \\ Alameda Urquijo s/n, 48013 Bilbao, Spain \\ ${ }^{* *}$ Centro de Tecnologías Aeronáuticas (CTA), Parque Tecnológico de Álava, Juan de la Cierva 1, 01510 Miñano, \\ Spain
}

by A. Mendioroz*, A. Salazar*, F. Alonso** and I. Sáez-Ocáriz**

In the last decade, a new type of excitation has been successfully introduced in the field of thermographic nondestructive evaluation (NDE) for the detection of defects such as cracks or delaminations: the so called vibrothermography or thermosonics [1,2]. In contrast to the more classical set-up in which the energy is delivered at the sample surface by optical means and the thermal waves propagate into the inner volume, in vibrothermographic arrangements the sample is mechanically excited by sonic or ultrasonic oscillations. The excitation is carried-out by coupling an ultrasonic transducer to the sample surface. In general, the propagation of the damped acoustic waves along the material converts mechanical energy into thermal energy, but in the vicinity of the defects the energy dissipation is bigger due to friction between the faces of the defect and/or stress concentration at the surrounding area. In metals, where the acoustical damping is relatively low, this mechanical excitation acts as a selective inner heat source, located at the defects, that diffuses inside the sample and can be detected as a temperature variation at its surface by means of an infrared (IR) camera.

As in optically excited IR thermography, two configurations have been mainly implemented in vibrothermography: one is the so-called lock-in vibrothermography [1] in which the high frequency oscillation is amplitude- (or frequency-) modulated at a much lower frequency and the detection lock-in system, synchronized with the amplitude varying input signal, detects the amplitude and phase of the surface temperature. The second configuration, called burst vibrothermography or sonic IR [2], consists of exciting the sample by a brief (typically 50-200 ms) ultrasonic pulse and detecting the surface temperature as a function of time, also by an IR camera. The main advantage of any of these mechanical excitations if compared to optical excitation is that in the latter case cracks are barely detectable, while vibrothermography has proven to be a useful technique for the detection of such defects in a wide variety of materials like metals, polymers and composites $[1,2,3]$. The reason for this is that in typical geometric arrangements of optically excited IR thermography, the incident light flux is perpendicular to the sample surface and so is the main heat flux inside the material. As cracks usually propagate perpendicular to the sample surface, the presence of these defects does not produce significant signatures at the sample's surface temperature. On the opposite, as mentioned above, mechanical excitation in vibrothermography "turns" the defect into a heat source, which makes it detectable.

Whatever configuration, either the modulated or the pulsed one is used, the physical processes contributing to the measured temperature variations at the sample surface after the initial mechanical excitation, are rather complex: the propagation of the acoustic wave inside the material, the mechanisms by which heat is generated at the defects, and the diffusion of this thermal energy to the sample's surface. Moreover, since the excitation method requires contact between the sample and the vibration generator, experimental issues related to the particular configuration of the piezoelectric transducer coupling, and the way the sample is held in place, introduce even more complexity to the task of understanding the whole process [3].

In this work we address the study of the final step in this process in the case of amplitude modulated lockin vibrothermography, i. e. the diffusion of the thermal energy generated at the defect to the surface, and its temperature distribution. We present simulations of the amplitude and phase of the oscillating temperature distribution at the sample surface induced by a crack together with experimental amplitude and phase data recorded for real cracked specimens. In particular, we have chosen metallic plates, of different thicknesses and materials as Titanium, steel and Aluminium.

Concerning the theoretical modelling of the temperature distribution, the basic geometry we have worked with is shown in figure 1 . The crack is considered as a non-continuous, size varying, flat heat source perpendicular to the sample surface, consisting of rectangles of dimensions $a_{i}, c_{i}$, separated by distances $s_{j}$, buried at different depths $d_{i}$ along its length, each with uniform heat generation. Since we are dealing with metallic plates whose thicknesses (about $10^{-3} \mathrm{~m}$ ) are smaller than the thermal diffusion length at the modulation frequencies $f$ we are working with (around $0.1 \mathrm{~Hz}$ ), we have used the images method to calculate the temperature at the sample surface, $z=0$.

We have performed the calculation by numerically integrating the contribution of all infinitesimal heat sources that configure our irregular crack together with their corresponding images, to the temperature distribution at the sample surface [4]:

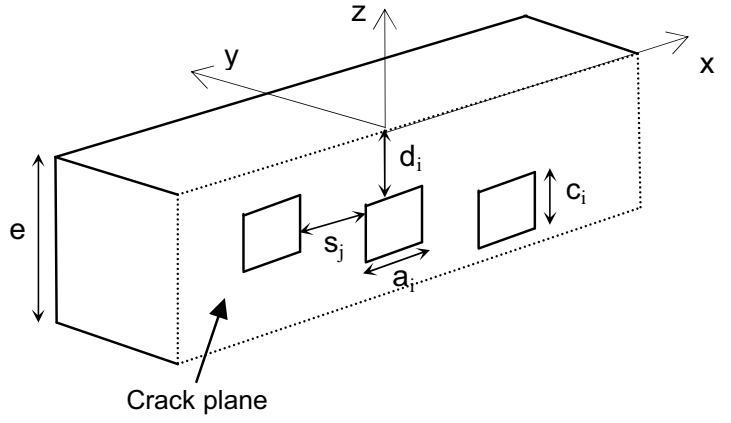

Fig. 1. In-plane view of the crack modeled for simulations. to the temperature distribution at the sample surface [4]: 


$$
T(x, y)=\iint_{\text {sources }} \frac{P_{o}}{2 \pi K} \frac{e^{-q \sqrt{(x-\alpha)^{2}+(y-\beta)^{2}+(z-\gamma)^{2}}}}{\sqrt{(x-\alpha)^{2}+(y-\beta)^{2}+(z-\gamma)^{2}}} d \alpha d \gamma .
$$

Here, $\alpha, \beta$, and $\gamma$ are the $\mathrm{x}, \mathrm{y}$, and $\mathrm{z}$ coordinates of the sources respectively, $q=\sqrt{i \omega / D}$ is the thermal wave vector, with $\omega=2 \pi \mathrm{f}, D$ and $K$ are the thermal diffusivity and conductivity of the sample respectively, and $P_{o}$ is the energy delivered per unit surface and unit time.

In order to compare the experimental results to the predictions of the model, here we show the theoretical isophases for a crack modelled as three identical $\left(a=2 \cdot 10^{-3} \mathrm{~m}, b=0.4 \cdot 10^{-3} \mathrm{~m}, d=0.55 \cdot 10^{-3} \mathrm{~m}\right)$, equally spaced $\left(\mathrm{s}=2 \cdot 10^{-3} \mathrm{~m}\right)$ sources, for modulation frequencies of $0.05 \mathrm{~Hz}$ and $0.5 \mathrm{~Hz}$., in a Titanium plate of $\mathrm{e}=10^{-3} \mathrm{~m}$ thickness. The results are shown in figure 2 , where the horizontal coordinate is $x(m)$ and the vertical is $y(m)$.

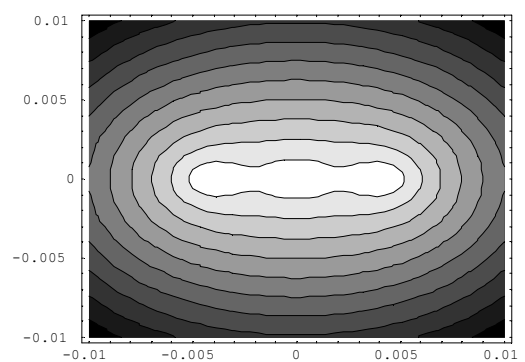

(a)

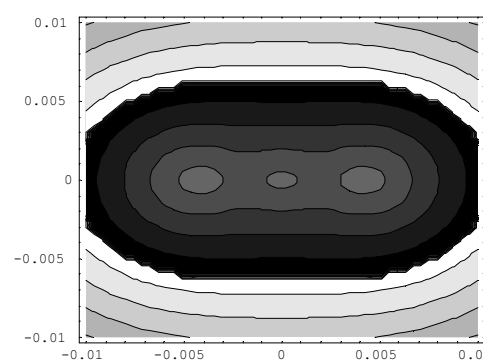

(b)

Fig. 2. Simulated temperature phase distribution at the surface of a $10^{-3} \mathrm{~m}$ thick Titanium plate at amplitude modulation frequency of (a) $0.05 \mathrm{~Hz}$ and (b) $0.2 \mathrm{~Hz}$.

As can be observed, for the higher frequency the isophases reproduce better the shape of the thermal sources, the lower frequency map looking smoother and with less resolution. However, at high enough modulation frequencies the thermal diffusion length might be short enough so that a defect at a certain depth is not detectable at the sample surface. Accordingly, there is an optimum modulation frequency for which the crack is detected with the best resolution.

We have recorded the phase thermograms of a plate made out of Titanium of thickness $10^{-3} \mathrm{~m}$, at 0.05 $\mathrm{Hz}$ and $0.2 \mathrm{~Hz}$, exhibiting a long partially open crack along its surface. The data is shown in figure 3.

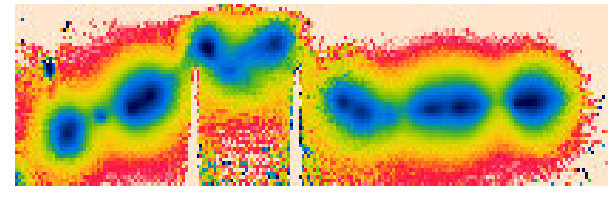

(a)

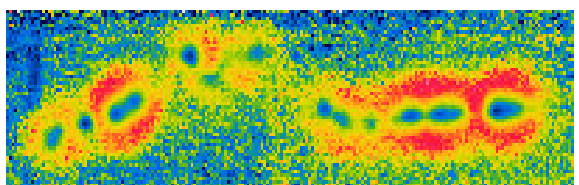

(b)

Fig. 3. Surface temperature phase distribution of a $10^{-3} \mathrm{~m}$ thick aluminum plate, after ultrasonic excitation at 20 $\mathrm{kHz}$, modulated at (a) $0.05 \mathrm{~Hz}$ and (b) $0.2 \mathrm{~Hz}$.

The experimental data also show a better resolution in the higher frequency image, in accordance with the theoretical results. On the other hand, the existence of "blind" regions could be related either to open crack, where friction between the two faces does not take place, or to the ultrasonic frequency chosen in the experiment, which might lead to the appearance of standing waves inside the sample, the dark regions corresponding to nodes or regions where the two edges of the crack move parallel to each other for the excited mode [5]. We intend to experimentally analyze the influence of both the ultrasound and the modulation frequency in the detection of calibrated cracks and to compare the experimental dependence on the modulation frequency to the predictions of an extended theoretical model including non flat cracks with non uniform heat generation. We hope that the results might contribute to better understand the capabilities of the technique to characterize this kind of defects.

This work has been supported by the Ministerio de Educación y Ciencia through research grant No MAT2005-02999.

\section{REFERENCES}

[1] J. Rantala, D. Wu, and G Busse. Amplitude-modulated lock-in vibrothermography for NDE of polymers and composites, Research in Nondestructive Evaluation, 7 (1996) 215-228.

[2] L. D. Favro, X. Han, Z. Ougan, G. Sun, H. Sua, and R. L. Thomas. Infrared imaging of defects heated by a sonic pulse, Review of scientific instruments, 71 (2000) 2418-2421

[3] L. D. Favro, X. Han, Z. Ouyang, G. Sun, and R. L. Thomas. Sonic IR imaging of cracks and delaminations, Analytical Sciences, (2001) 451-453.

[4] H. S. Carslaw, and J.C. Jaeger. Conduction of heat in solids. Oxford University Press (1959).

[5] A. Gleiter, C. Spiessberg, and G. Busse. Improved ultrasound activated thermography using frequency analisys, QUIRT Journal, 4 (2007) 155-164. 